\title{
A Novel Control Method of Three-Level Converter Neutral-Point Potential Balance
}

\author{
X.H. Chai, C.J. Zhang, B. Ben, Z.N. Guo \\ School of Electrical Engineering \\ Yanshan University \\ China
}

\begin{abstract}
With the neutral-point-clamped (NPC) converter widely applied to direct-drive wind energy generation system, wheather to guarantee the dc-link neutral-point potential (NPP) balance has a crucial influence on the system. Research on gridside NPC three-level converter, firstly, this paper established NPP fluctuation mathematical model, analyzed the mechanism of NPP AC-DC fluctuations, deduced the relationship between NPP error calculation and system operation mode (rectifier-mode or inverter-mode), and established NPP control model. Secondly, the inherent law of NPP balancing control and the controller parameter tuning method were given by theoretical analysis. In order to suppress NPP AC fluctuations at the extreme, this paper proposes a method designing controller parameters according to the largest open-loop cutoff frequency by system frequency characteristic analysis. The NPP control method has the following advantages: effectively suppressing the NPP AC-DC fluctuations at the same time, fast dynamic response of positive and negative dc-link voltage and high utilization rate of DC voltage. Finally, the correctness of the theoretical analysis was verified by MATLAB simulation.
\end{abstract}

Keywords-three-level converter; neutral-point potential; mathematical model; cutoff frequency

\section{INTRODUCTION}

In order to meet the need of direct-drive wind power generation system full power converter, the NPC converteris, which has the advantages of small switch voltage stress, low harmonic content and small switching losses of power device, has been widely used in direct-drive wind power generation system.

The NPP offset will increase the voltage stress of the device, easily cause the device overvoltage damage, especially cause system unstable operation [1], therefore, whether dc-link NPP is balanced has a crucial effect on the performance of NPC converter [2].The dc-link NPP fluctuation is divided into two kinds: $\mathrm{AC}$ fluctuation and DC fluctuation. How to eliminate the NPP AC-DC fluctuation has been studied in many literatures. In [3], using the zero-sequence voltage real-time calculation to realize zero-sequence injection can suppress the NPP AC-DC fluctuation simultaneously, but the calculation is more complicated. Based on the Space Vector Modulation (SVM), NPP balance control is realized by adjusting the vector time [4]. Due to the advantage of high voltage utilization ratio and vector selection flexibility, etc., SVM is widely applied, but needs large amount of calculation. In [5], the NPP AC fluctuation is eliminated through injection three times harmonic current component.
Based on the three-level SPWM, this paper proposes a NPP control method based on the NPP control loop, the basic principle is that the NPP fluctuation is effectively restrained by the superposition of the NPP control loop output zero-sequence voltage to three-phase modulation voltage. The control principle of this method is similar to the zero-sequence voltage injection method proposed, but does not require the extra zero-sequence component generating circuit, meanwhile is intrinsic consistent with NPP control method based on SVPWM, but the proposed method is simple and easy to realize.

\section{SySTEM TOPOLOGY AND MATHEMATICAL MODEL}

In direct-drive permanent magnet (PM) wind power generation system, the purpose of grid side converter (GSC) is to stabilize dc-link voltage. For the DC side, the circuit equations of dc side are shown as:

$$
C \frac{\mathrm{d} \Delta u_{\mathrm{dc}}}{\mathrm{dt}}=-i_{\mathrm{dc} \_\mathrm{GSG}+}+i_{\mathrm{dc} \_\mathrm{GSG}-}
$$

where, $i_{\mathrm{dc} \_ \text {GSG+ }}$ and $i_{\mathrm{dc} \_ \text {GSG- }}$ are the grid-side currents through the positive dc-link and the negative dc-link, respectively. $C$ is the DC capacitor.

The expressions of $i_{\mathrm{dc} \_\mathrm{GSG}+}$ and $i_{\mathrm{dc} \_\mathrm{GSG}-}$ are as follows:

$$
i_{\mathrm{dc} \_\mathrm{GSG}+}=i_{\mathrm{a}+}+i_{\mathrm{b}+}+i_{\mathrm{c}+} \quad i_{\mathrm{dc} \_\mathrm{GSG}-}=i_{\mathrm{a}-}+i_{\mathrm{b}-}+i_{\mathrm{c}-}
$$

where, $i_{\mathrm{x}_{+}}, i_{\mathrm{x}-}$ are the currents through upper bridge arm and lower bridge arm respectively. subscript $\mathrm{x}=\mathrm{a}, \mathrm{b}, \mathrm{c}$;

According to the characteristic of NPC circuit, it can be obtained as:

$$
i_{\mathrm{x}+}=\left\{\begin{array}{cc}
s_{\mathrm{x}} i_{\mathrm{xl}} & u_{\mathrm{xr}} \geq 0 \\
0 & u_{\mathrm{xr}}<0
\end{array} \quad i_{\mathrm{x}-}=\left\{\begin{array}{cc}
0 & u_{\mathrm{xr}} \geq 0 \\
s_{\mathrm{x}} i_{\mathrm{xl}} & u_{\mathrm{xr}}<0
\end{array}\right.\right.
$$

where, $i_{\mathrm{xl}}$ is the inductor current; $u_{\mathrm{xr}}$ is the modulation voltage.

Substituting (2) and (3) into (1), then the result is averaged, the average mathematical model of dc-side is shown as:

$$
C \frac{\mathrm{d} \Delta u_{\mathrm{dc}}}{\mathrm{dt}}=-\left(\beta_{\mathrm{a}} \cdot \operatorname{sgn}\left(\beta_{\mathrm{a}}\right) i_{\mathrm{al}}+\beta_{\mathrm{b}} \cdot \operatorname{sgn}\left(\beta_{\mathrm{b}}\right) i_{\mathrm{bl}}+\beta_{\mathrm{c}} \cdot \operatorname{sgn}\left(\beta_{\mathrm{c}}\right) i_{\mathrm{cl}}\right)
$$


where, $\beta_{\mathrm{x}}=<s_{\mathrm{x}}>_{T_{\mathrm{s}}}=u_{\mathrm{xr}} / V_{\mathrm{cm}}$ is the average of the switching function; $V_{\mathrm{cm}}$ is the amplitude of the triangular carriers.

\section{THE MATHEMATICAL MODEL OF NPP FLUCTUATIONS}

Substituting $\beta_{\mathrm{x}}=\beta_{\mathrm{x}}^{\prime}+\beta_{\mathrm{o}}$ into (1), it can be obtained as:

$$
\begin{aligned}
C \frac{\mathrm{d} \Delta u_{\mathrm{dc}}}{\mathrm{dt}}= & -\left[\beta_{\mathrm{a}}^{\prime} \cdot \operatorname{sgn}\left(\beta_{\mathrm{a}}\right) i_{\mathrm{al}}+\beta_{\mathrm{b}}^{\prime} \cdot \operatorname{sgn}\left(\beta_{\mathrm{b}}\right) i_{\mathrm{bl}}+\beta_{\mathrm{c}}^{\prime} \cdot \operatorname{sgn}\left(\beta_{\mathrm{c}}\right) i_{\mathrm{cl}}\right] \\
& -\left[\operatorname{sgn}\left(\beta_{\mathrm{a}}\right) i_{\mathrm{al}}+\operatorname{sgn}\left(\beta_{\mathrm{b}}\right) i_{\mathrm{bl}}+\operatorname{sgn}\left(\beta_{\mathrm{c}}\right) i_{\mathrm{cl}}\right] \beta_{0}
\end{aligned}
$$

Assuming $\Delta u_{\mathrm{dc}}=0$, from (5) it can be written as

$$
\beta_{0}^{\prime}=-\frac{\beta_{\mathrm{a}}^{\prime} \operatorname{sgn}\left(\beta_{\mathrm{a}}\right) i_{\mathrm{al}}+\beta_{\mathrm{b}}^{\prime} \operatorname{sgn}\left(\beta_{\mathrm{b}}\right) i_{\mathrm{bl}}+\beta_{\mathrm{c}}^{\prime} \operatorname{sgn}\left(\beta_{\mathrm{c}}\right) i_{\mathrm{cl}}}{\operatorname{sgn}\left(\beta_{\mathrm{a}}\right) i_{\mathrm{al}}+\operatorname{sgn}\left(\beta_{\mathrm{b}}\right) i_{\mathrm{bl}}+\operatorname{sgn}\left(\beta_{\mathrm{c}}\right) i_{\mathrm{cl}}}
$$

When the dc-link voltage balance, $\beta_{\mathrm{x}}^{\prime}=\beta_{\mathrm{x}}$. Substituting $i_{\mathrm{al}}, i_{\mathrm{bl}}, i_{\mathrm{cl}}$ and $\beta_{a}^{\prime}, \beta_{\mathrm{b}}^{\prime}, \beta_{\mathrm{c}}^{\prime}$ into (1), zero-sequence component under ideal condition can be derived as:

$$
\beta_{0}^{\prime}=\pi M \sin (3 \theta) / 12
$$

where, $M$ is the modulation ratio. From (7), it can be known that dc-link NPP contains ac fluctuations which is three times the fundamental frequency of grid voltage (Abbreviated as there times frequency) in the ideal condition.

Sum

$$
\beta_{0}=\beta_{0}^{\prime}+\Delta \beta_{0}
$$

Substituting (8) and (6) into (1) leads to

$$
C \frac{\mathrm{d} \Delta u_{\mathrm{dc}}}{\mathrm{dt}}=-\left[\operatorname{sgn}\left(\beta_{\mathrm{a}}\right) i_{\mathrm{al}}+\operatorname{sgn}\left(\beta_{\mathrm{b}}\right) i_{\mathrm{bl}}+\operatorname{sgn}\left(\beta_{\mathrm{c}}\right) i_{\mathrm{cl}}\right] \Delta \beta_{0}
$$

Due to the filter inductance value is very small, inductive voltage drop can be negligible. So from (9) the mathematical model of NPP fluctuation under two operation modes is as follows:

$$
C \frac{\mathrm{d} \Delta u_{\mathrm{dc}}}{\mathrm{dt}}=\left\{\begin{array}{cc}
\Delta \beta_{0}\left(\left|i_{\mathrm{al}}\right|+\left|i_{\mathrm{bl}}\right|+\left|i_{\mathrm{cl}}\right|\right) & \text { rectifier } \\
-\Delta \beta_{0}\left(\left|i_{\mathrm{al}}\right|+\left|i_{\mathrm{bl}}\right|+\left|i_{\mathrm{cl}}\right|\right) & \text { inverter }
\end{array}\right.
$$

Ignore all harmonic components, the average of $\left(\left|i_{\mathrm{al}}\right|+\left|i_{\mathrm{bl}}\right|+\left|i_{\mathrm{cl}}\right|\right) / 2$ is $3 I_{\mathrm{M}} / \pi$, where, $I_{\mathrm{M}}$ is phase-current amplitude. When using the voltage oriented vector control, the system is operating at rectifier-mode, $I_{\mathrm{M}}=-\sqrt{2 / 3} I_{\mathrm{dl}}\left(I_{\mathrm{dl}}<0\right)$; When the system is operating at inverter-mode, $I_{\mathrm{M}}=\sqrt{2 / 3} I_{\mathrm{dl}} \quad\left(I_{\mathrm{dl}}>0\right)$, mathematical model of DC NPP fluctuations under synchronous rotating coordinates is achieved as:

$$
C \frac{\mathrm{d} \Delta u_{\mathrm{dc}}}{\mathrm{dt}}=-\frac{2 \sqrt{6}}{\pi} I_{\mathrm{dl}} \Delta \beta_{0}
$$

\section{THE DESIGN OF THE NPP BALANCE CONTROL SYSTEM}

\section{A. The Strategy of Neutral-Point Potential Control}

In order to suppress the NPP fluctuations, the NPP control strategy based on NPP control loop is adopted, the output of NPP control loop is added to the three-phase modulation voltage, the control principle is shown in Figure.1.

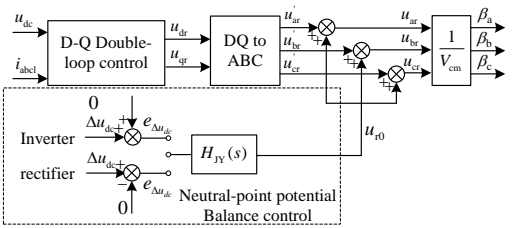

FIGURE I. .PRINCIPLE DIAGRAM OF THE SYSTEM CONTROL.

\section{B. The Controller Parameters Design}

According to (11), the model of NPP fluctuation is established, shown in Figure.2.

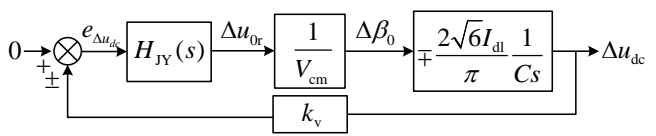

FIGURE II. NPP FLUCTUATION CONTROL MODEL.

Where, $k_{\mathrm{v}}$ is the sampling coefficient.

In order to improve the system anti-interference ability, the controller adopts single zero double pole controller, the transfer function is as:

$$
H_{\mathrm{JY}}(s)=k_{\mathrm{pjy}} \frac{T_{\mathrm{zjy}} s+1}{T_{\mathrm{zjy}} s\left(T_{\mathrm{ov}} s+1\right)}
$$

Where, $k_{\mathrm{pjy}}$ is the proportional gain; $T_{\mathrm{zjy}}$ is zero time-constant; $T_{\mathrm{ov}}$ is the filter time-constant.

The open-loop transfer function of NPP control loop is

$$
G_{\mathrm{YJO}}(s)=\frac{k_{\mathrm{jy}}\left(T_{\mathrm{zjy}} s+1\right)}{s^{2}\left(T_{\mathrm{ov}} s+1\right)}
$$

where, $k_{\mathrm{jy}}=2 \sqrt{6} I_{d} k_{\mathrm{pjy}} k_{\mathrm{v}} /\left(\pi T_{\mathrm{zjy}} V_{\mathrm{cm}} C\right)$ is open-loop gain.

Assuming that filter pole $1 / T_{\mathrm{ov}}$ have been identified, according to the typical II system design, the controller parameter tuning formula is as follows:

$$
\left\{\begin{array}{l}
T_{\mathrm{zjy}}=h T_{\mathrm{ov}} \\
k_{\mathrm{pjy}}=\frac{\pi V_{\mathrm{cm}} C(h+1)}{4 \sqrt{6} h T_{\mathrm{ov}} I_{d} k_{\mathrm{v}}}
\end{array}\right.
$$


Considering the system performance of following and antiinterference, the intermediate frequency width $h=5$ is a good choice.

The premise of the parameter tuning method is that the controller filter poles $1 / T_{\mathrm{ov}}$ is known, so how to select $T_{\mathrm{ov}}$ is the key point in the controller parameter tuning, and has crucial influence on system performance. When $T_{\mathrm{ov}}$ is different, it has no impact on the type of system dynamic response, phase angle stability margin and overshoot, however, it has a great impact on the system dynamic response speed, high frequency anti-interference ability and the system gain. This paper mainly analyzes the influence of the proposed method on three times frequency $\left(\omega_{3 h}\right)$ signal, setting the gain of three times frequency signal is $k_{3 \mathrm{~h}}$.

As $1 / T_{\text {ov }}$ (cutoff frequency or bandwidth) increases, the system amplitude-frequency characteristics moves to the right, $k_{3 \mathrm{~h}}$ increases, three times frequency steady-state difference of NPP decreases. Therefore, in order to reduce the NPP AC fluctuation, the cutoff frequency of NPP control loop should be increased as much as possible. The controller output is superimposed on the modulation voltage directly, and the control strategy is based on the mathematical model of switching cycle average, so the largest open-loop cutoff frequency is half of the switching frequency. Because of the limitation of the maximum open-loop cutoff frequency, $k_{3 \mathrm{~h}}$ can't increase without limitation. So three times frequency zero-sequence component of the NPP cannot be completely eliminated, but it can be controlled to be very small, basically negligible.

\section{Simulation}

The MATLAB/Simulink parameters are as follow: the amplitude of grid voltage is $311 \mathrm{~V}$; the switching frequency is $10 \mathrm{kHz} . L_{\mathrm{f}}=5 \mathrm{mH} ; C=1410 \mu \mathrm{F} ; u_{\mathrm{dc}}=850 \mathrm{~V}$.

\section{A. Simulation without NPP Control Loop}

At $\mathrm{t}=0.2 \mathrm{~s}$, the system switches from balanced-load to unbalanced-load. Because of no NPP control, the positive and negative dc-link voltage has differential, as Figure. 3 shown, however $u_{\mathrm{dc}}$ remained stable at $850 \mathrm{~V}$ constant.

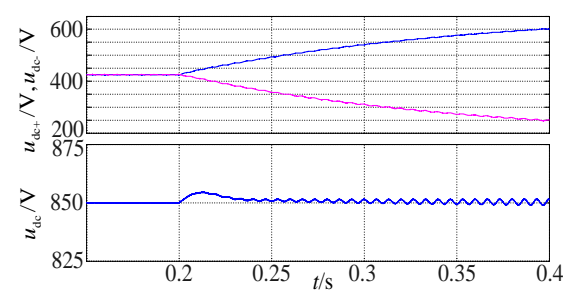

FIGURE III. DC-LINK VOLTAGE WAVEFORM WITHOUT NPP CONTROL LOOP.

\section{B. Mode-Switching between Inverter-Mode and Rectifier - Mode}

At the startup stage, GSC operates in rectifier-mode to charge for dc side capacitor. At $t=0.2 \mathrm{~s}$, the system switches from balanced-load to unbalanced-load. When $t=0.4 \mathrm{~s}$, the
MSC puts into the system and runs in MPPT mode, the GSC switch from rectifier-mode to inverter-mode, and the power is delivered from the PMSG to the grid, as Figure.4 shown.

When $e_{\Delta v_{d c}}=-\Delta u_{\mathrm{dc}}$, the system is stable in rectifier-mode, but after switches to inverter-mode, unstable because that NPP control change from negative feedback to positive feedback, simulation result is shown is Figure.4(a). In order to guarantee the system stable operation, the calculation of $e_{\Delta v_{d c}}$ need to vary with the operation mode, simulation wave is shown in Figure.4(b).

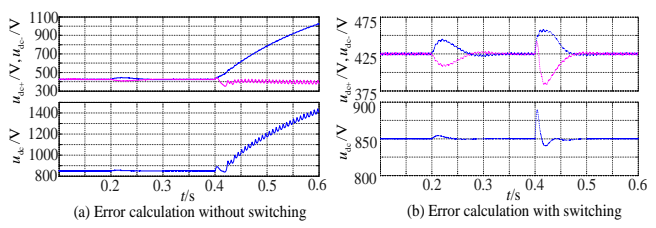

FIGURE IV. SIMULATION WAVEFORMS OF SYSTEM SWITCHING IN TWO WORKING STATES.

\section{Comparison of Different Controller Parameters}

Different control parameters have a great influence on the NPP control, the simulation results are as follow:

(1) When $1 / T_{\mathrm{ov}}$ is half of the fundamental frequency, According to the frequency characteristic we can know $k_{3 \mathrm{~h}}<1$, the system can't effectively suppress PNN AC fluctuation. As shown in Figure.5 the three-phase modulation voltage approximates sinusoidal, the positive and negative dc-link voltage, which is balanced, contains $\omega_{3 \mathrm{~h}} \mathrm{AC}$ fluctuation, and the dynamic response is very slow.

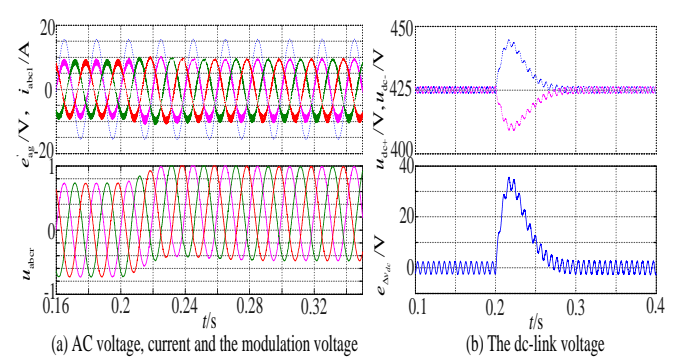

FIGURE V. SIMULATION WAVEFORMS WITH NPP CONTROL LOOP.

(2) The NPP control loop controller parameters is designed according to the largest open-loop cutoff frequency, $1 / T_{\mathrm{ov}}=16667 \pi$ and $k_{\mathrm{pjy}}=3792$, the simulation results are shown in Figure.6.
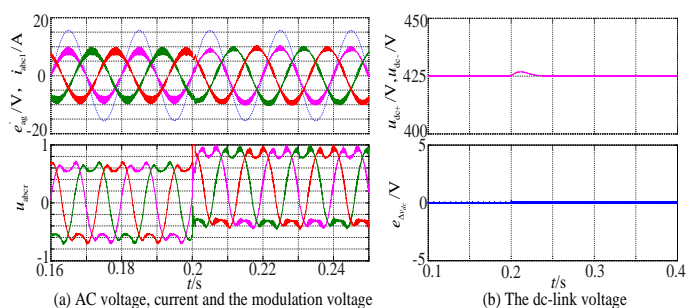

FIGURE VI. SIMULATION WAVEFORMS WITH NPP CONTROL LOOP. 
The results show: Firstly, this control method is Equivalent to injecting three times harmonic to three-phase modulation voltage, is similar to SVPWM, and the voltage utilization is improved; Secondly, the positive and negative dc-link voltage is balanced and no ac fluctuation; Thirdly, the dynamic response of the dc-link is fast.

\section{CONCLUSION}

A new control method to suppress the NPP fluctuation is proposed, and this method is verified by grid-side NPC converter based on the permanent direct wind generation system. Additional, the detail mathematical model of the NPP control loop is established and the influence of controller parameters is analyzed.

From the model of the control loop, the operation mode can be divided into two types: rectifier-mode and invertermode. In rectifier-mode, the error equation is $e_{\Delta v_{d c}}=-\Delta u_{\mathrm{dc}}$, while in inverter-mode the error equation is $e_{\Delta v_{d c}}=\Delta u_{\mathrm{dc}}$. When the controller is desighed according to largest open-loop cutoff frequency principle, the dc and ac NPP fluctuation can be suppressed simultaneously. Finally, the proposed method has following advantage: Control algorithm is simple, dynamic response of dc-link is fast, and voltage utilization ratio is high.

\section{ACKNOWLEDGEMENTS}

This work was supported by National Natural Science Foundation (51477148) and Natural Science Foundation of Hebei Province of China (E2013203380).

\section{REFERENCES}

[1] B. Wu, High-Power Converters and AC Drives. Hoboken, NJ, USA: Wiley, 2006.

[2] Weidong Jiang, Qun-jing Wang, Xiao-feng Shi, et al., Low Frequency Oscillation of Neutral-point potential of Neutral-point-clamped Threelevel VSI Under SVPWM Control. Proceedings of the CSEE, 29(3), pp. 19-55, 2009.

[3] J. Pou, D. Boroyevich, R. Pindado, Effects of imbalances and nonlinear loads on the voltage balance of a neutral-point-clamped inverter. IEEE Trans. Power Electron, 20(1), pp. 123-131, 2005.

[4] Yongqing Meng, Chuanwen Shen, Zheng Liu, et al. Study on neutralpoint potential control method for three-level NPC rectifiers by injecting zero-sequence voltage. Proceedings of the CSEE, Vol. 27, No. 10, pp. 92-100, Apr. 2007.

[5] Amit Kumar Gupta, Ashwin M. Khambadkone, A Simple Space Vector PWM Scheme to Operate a Three-Level NPC Inverter at High Modulation Index Including Overmodulation Region With Neutral Point Balancing. IEEE TRANSACTIONS ON INDUSTRY APPLICATIONS, 43(3), pp. 751-759, 2007. 